\title{
DeepHist: Towards a Deep Learning-based Computational History of Trends in the NIPS
}

\author{
Amna Dridi \\ Birmingham City University, UK \\ Email: amna.dridi@mail.bcu.ac.uk \\ Jagdev Bhogal \\ Birmingham City University, UK \\ Email: jagdev.bhogal@bcu.ac.uk
}

\author{
Mohamed Medhat Gaber \\ Birmingham City University, UK \\ Email: mohamed.gaber@bcu.ac.uk
}

\author{
R. Muhammad Atif Azad \\ Birmingham City University, UK \\ Email: atif.azad@bcu.ac.uk
}

\begin{abstract}
Research in analysis of big scholarly data has increased in the recent past and it aims to understand research dynamics and forecast research trends. The ultimate objective in this research is to design and implement novel and scalable methods for extracting knowledge and computational history. While citations are highly used to identify emerging/rising research topics, they can take months or even years to stabilise enough to reveal research trends. Consequently, it is necessary to develop faster yet accurate methods for trend analysis and computational history that dig into content and semantics of an article. Therefore, this paper aims to conduct a fine-grained content analysis of scientific corpora from the domain of $\mathrm{Ma}$ chine Learning. This analysis uses DeepHist, a deep learningbased computational history approach; the approach relies on a dynamic word embedding that aims to represent words with low-dimensional vectors computed by deep neural networks. The scientific corpora come from 5991 publications from Neural Information Processing Systems (NIPS) conference between 1987 and 2015 which are divided into six 5-year timespans. The analysis of these corpora generates visualisations produced by applying t-distributed stochastic neighbor embedding (t-SNE) for dimensionality reduction. The qualitative and quantitative study reported here reveals the evolution of the prominent Machine Learning keywords; this evolution supports the popularity of current research topics in the field. This support is evident given how well the popularity of the detected keywords correlates with the citation counts received by their corresponding papers: Spearman's positive correlation is $100 \%$. With such a strong result, this work evidences the utility of deep learning techniques for determining the computational history of science.
\end{abstract}

\section{INTRODUCTION}

The abundance of scholarly data sources like digital libraries and academic social networks has enabled big scholarly data analysis. With this data it is now possible to develop a computational history of science and consequently ask interesting questions such as what scientific trends are emerging in a given scientific field.

Many researchers have realised the importance of mining scholarly data to understand the dynamics of science. Generally, tracking the dynamics of science is highly related to revealing hidden trends within the vast quantity of available resources. Hence, trend analysis has been popular in the research community of scholarly data analysis [1] that looks for popular keywords.
Commonly the detection of evolving scientific keywords or trends has widely relied on citation analysis; however, this analysis is not fool proof. While citation counts can signify the importance of scientific work, these counts may also grow for non-scientific reasons [2]. Instead, there can be other interesting papers - termed as sleeping beauties [3] - which do not get cited much for several years after publication, but then unexpectedly start getting cited.

For the reasons cited above and the fact that citationbased approaches fail to dig into the paper content which could lead to a more accurate trend detection, some emerging researchers [4], [5] have followed a particular direction in computational history which is the use of topic models to analyse the rise and fall of research topics and accordingly the progress of science.

Going beyond the topic models that capture document level associations between words, our aim in this paper is to learn word embeddings across time in scientific corpora due to the ability of word embeddings to detect very local associations. For instance, it has been shown that word embeddings can successfully capture both the semantic and the syntactic features of words [6]. They can complement topic models, or stand alone as an approach to building a computational history of science.

To do so, this paper introduces DeepHist, a deep-learning based approach to computational history that uses dynamic word embedding in order to study the semantic shifts of words and consequently detect the evolving scientific keywords; in this paper, words and keywords are used interchangeably. To detect the semantic shift of words, we build a similarity matrix that records similarity between frequent keywords embedding vectors over each timespan. Then, based on these matrices, we create an acceleration matrix that computes the acceleration of various keywords over subsequent timespans in order to detect fast converging keywords. The acceleration represents the difference in similarities between keywords over two successive timespans. The subject area used in this work is Machine Learning. This choice owes both to the authors' background in this subject area, and the fact that Machine Learning has enjoyed notable successes in the recent years.

We experiment and evaluate our proposed approach with 
5991 publications from the NIPS conference proceedings between the years 1987 and 2015 that we divided into six timespans of 5-years each. For evaluating the effectiveness of our embeddings in detecting emerging scientific keywords, we adopt both qualitative and quantitative methods.

- Qualitatively, we illustrate the advantages of dynamic embeddings that show the evolving scientific keywords in the area of Machine Learning for each timespan by plotting keyword vectors after reducing their dimensions with t-distributed stochastic neighbor embedding ( $t$-SNE) [7]. All t-SNE visualisations show that our embeddings are able to illustrate the fast acceleration between the emerging scientific keywords.

- Quantitatively, to validate our approach against the commonly used citation analysis approach, we collect the citation counts of NIPS publications having detected the emerging keywords in their titles, track the citation evolution over time and compute its correlation with the obtained similarities captured by our embeddings. The experimental results show the effectiveness of our approach that was able to detect that the keywords "neural" and "learning" were getting similar across time and rising by $70 \%$ in similarity in the timespan 2007-2011 when the emerging keyword "deep learning" started to flourish as a neural-based learning. Interestingly, our approach succeeded to achieve a $100 \%$ positive correlation between the citation counts and the similarities returned by our embeddings.

The rest of the paper is organised as follows. Section II details our DeepHist methodology and how we employ word embeddings, namely skip-gram model to detect rising scientific keywords in the area of Machine Learning. Section III describes the NIPS dataset we have used, presents and discusses the obtained results. A summary of existing work is briefly presented in Section IV. Finally, in section $\mathrm{V}$ we conclude the paper and draw future directions.

\section{DEEPHIST}

This study introduces a deep-learning based computational history (DeepHist) that tracks rise or evolution of scientific keywords and hence the evolution of Machine Learning itself. Accordingly, we adopt a dynamic word embeddings technique to learn word vectors in a temporal fashion, in order to capture words that get geometrically closer, and hence reveal emerging keywords. The skip-gram $(S G)$ neural network architecture of word2vec embedding model [8] is used in this paper as it consistently performed better than the continuous bag of words $(\mathrm{CBOW})$ architecture.

\section{A. Skip-Gram Neural Network Model}

To learn high-quality distributed vector representations, the skip-gram (SG) neural network model was introduced by Mikolov et al. [6]; SG can successfully capture both the semantic and the syntactic word regularities [6].
1) Notation: We consider the corpora of NIPS publications collected across time (1987-2015). Formally, $P=\left(P_{1}, \ldots, P_{T}\right) \quad$ where each $P_{t} \mid t=1, \ldots, T$ is the corpus of all publications in the $t^{\text {th }}$ timespan, and $V=\left(\operatorname{word}_{1}, \ldots, \operatorname{word}_{N}\right)$ is the vocabulary that consists of $N$ words present in the corpora $P$ at any point in time; thus, it is likely for some word $_{i} \in V$ to not appear at all in some $P_{t} . V$ comprises of both emerging and dying words as they typically occur in scientific corpora.

Given this time-tagged scientific corpora, our goal is to find a dense, low-dimensional vector representation $u_{\text {word }_{i}}(t) \in R^{d}, d \ll|V|$ for each word word $_{i} \in V$ and each timespan $t=1, \ldots, T ; d$ is the dimensionality or the length of the word vectors. $\mathscr{M}_{\text {input }}$ is the matrix of size $N \times d$ that represents the input to hidden layer connections with each row representing a vocabulary word word $_{i}, i=1, \ldots, N$, and $\mathscr{M}_{\text {output }}$ is the matrix of size $d \times N$ that describes the connections from hidden layer to output layer where each column of $\mathscr{M}_{\text {output }}$ represents a word word $_{i} \in V$.

2) Model: Skip-gram model uses a single hidden layer, fully connected neural network as simplified in Figure 1

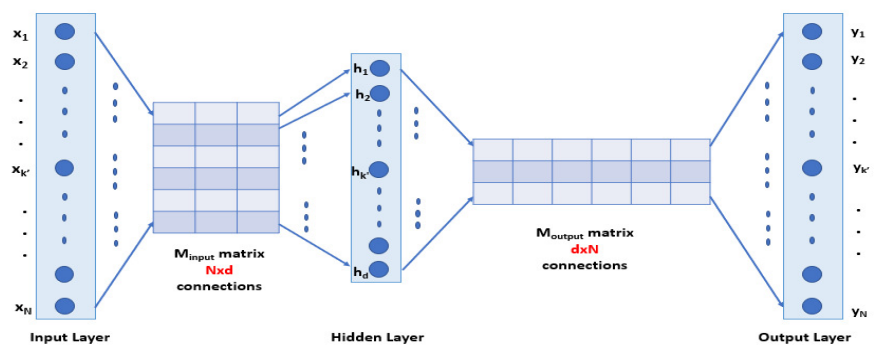

Fig. 1: Architecture of Skip-Gram Neural Network Model

The neurons in the hidden layer are all linear neurons. The input layer is set to have as many neurons as there are words in the vocabulary, i.e., $N$. The hidden layer size is set to the dimensionality $d$ of the resulting word vectors. The size of the output layer is same as the input layer and they are represented respectively by the matrices $\mathscr{M}_{\text {input }}|N \times d|$ and $\mathscr{M}_{\text {output }}|d \times N|$. The input to the network is encoded using " 1 -out of - $N$ " representation, meaning that only one input line is set to 1 and the rest of the input lines are set to zero.

The main idea of skip-gram is to predict the context given a word word $_{i}$. Note that the context is a window around word $d_{i}$ of maximum size $L$ that represents the span of words in the text; the span runs both backwards and forwards when iterating through the words during model training.

Since the goal of skip-gram model is to produce probabilities for the words in a context (context words) in the output layer given a word word $_{i}, \operatorname{Prob}\left(\text { word }_{\text {context }} \mid \operatorname{word}_{i}\right)_{i=1, \ldots, N}$, the model needs the neuron outputs in the output layer to sum to one. Word2vec achieves this by converting activation values of output layer to probabilities using the softmax function. Thus, the output of the $k^{\prime}$-th neuron is computed by the following expression:

$y_{k^{\prime}}=\operatorname{Prob}\left(\right.$ word $\left._{\text {context }} \mid \operatorname{word}_{i}\right)=\frac{\exp \left(\operatorname{activation}\left(k^{\prime}\right)\right)}{\sum_{n=1}^{N} \exp (\operatorname{activation}(n))}$ 


\section{B. Skip-Gram Hyperparametrisation}

Skip-Gram model depends on several hyperparameters; some of them crucially impact the quality of embeddings, especially vector dimensionality and context window. Despite that, the majority of applications that used word embeddings as features computed their vector representations with a default or arbitrary choice of hyperparameters.

Since the optimal hyperparameters values are known to be often data and task dependent, we proposed a domainspecific approach to hyperparametrisation [9] for skip-gram; the domain is scientific and specifically concerns Machine Learning literature. The approach uses the stability of $k$ nearest neighbors $(k-N N)$ of word vectors as the objective to measure while learning word2vec hyperparameters.

Stability is an important aspect of a learning algorithm. It has been widely used in clustering problems [10] to assess the quality of a clustering algorithm. Also, it has been applied in high-dimensional regression [11] for training parameter selection. Since word embedding produces high-dimensional word representations that can be organised into word clusters, we applied the $k$-NN algorithm to tune the hyperparameters of skip-gram model until the $k$-NN clustering is stable. $k$-NN clusters similar words based on their cosine similarities.

The basic idea of word embedding stability is the following: the quality of embedding inevitably depends on tuning hyperparameters defined previously, namely vector dimensionality and context window. If we choose accurate values of the tuning hyperparameters, then we expect to find $k$ words from different embeddings that are similar to a target word $\operatorname{word}_{i}$.

The $k$-NN embedding stability approach follows four steps:

1) Fix one hyperparameter at the beginning (choose the default value for example).

2) Tune the second hyperparameter by trying different values and training the model for each value.

3) Compute word similarities after each training and define $k$-nearest neighboring words.

4) Repeat steps 2 and 3 for the second hyperparameter to be tuned after fixing the first one (step 1) to the optimal value already obtained.

The $k$-NN stability is defined as the overlap rate of similar words resulting from two different embeddings.

$$
\text { stability }=\frac{\mathcal{S}_{E_{h}}^{\text {word }}{ }^{i} \cap \mathcal{S}_{E_{h^{\prime}}}^{\text {word }} d_{i}}{k} \times 100
$$

where $\mathcal{S}_{E_{h}}$ and $\mathcal{S}_{E_{h^{\prime}}}$ are two sets of words that are similar to a target word word $_{i}$ but were produced from two different embeddings $E_{h}$ and $E_{h^{\prime}}$ with different hyperparameter values. $k$ is the number of nearest neighbors to $\operatorname{word}_{i}$ given by the cosine similarity. In this study, $k$ is set to 5 . This choice is motivated by our aim to keep the word similarities as finegrained as possible in order to evaluate the quality of skipgram model within scientific text.

In our previous work [9], we showed that the optimal hyperparameters values are 200 and 6 for vector dimensionality $d$ and the context window respectively for the NIPS corpora we are using. Therefore, the skip-gram model is tuned with these hyperparameters in this work.

\section{Dynamic Skip-Gram Model}

To track the dynamism of skip-gram embeddings and measure the accelerations of potential emerging keywords, we propose to create a similarity matrix $M_{i j}(t)$ of size $\left|V^{\prime}\right| \times\left|V^{\prime}\right|$, $V^{\prime} \subset V$, for each timespan $t$ that corresponds to the distance metric between two words. Note that $V^{\prime}$ represent the set of frequent keywords. All distances between two words wor $_{i}$ $\left(w_{i}\right)$ and $\operatorname{word}_{j}\left(w_{j}\right)$ are calculated by the cosine similarity between embedding vectors $u_{w_{i}}$ and $u_{w_{j}}$. Recall that $M_{i j}(t)$ is a symmetric matrix.

$$
\operatorname{sim}\left(w_{i}, w_{j}\right)=\operatorname{cosine}\left(u_{w_{i}}, u_{w_{j}}\right)=\frac{u_{w_{i}} \cdot u_{w_{j}}}{\left\|u_{w_{i}}\right\| \cdot\left\|u_{w_{j}}\right\|}
$$

Then, we generate an acceleration matrix $A_{i j}$ of size $\left|V^{\prime \prime}\right| \times\left|V^{\prime \prime}\right|, V^{\prime \prime}=V_{t}^{\prime} \cap V_{t+1}^{\prime}$, that corresponds to the acceleration between two words $w_{i}$ and $w_{j}$ from $t$ to $t+1$. The acceleration between two words $w_{i}$ and $w_{j}$ acceleration $\left(w_{i}, w_{j}\right)$ is defined as follows.

$$
\operatorname{acceleration}\left(w_{i}, w_{j}\right)=\operatorname{sim}\left(w_{i}, w_{j}\right)^{t+1}-\operatorname{sim}\left(w_{i}, w_{j}\right)^{t}
$$

Two keywords are defined as emerging keywords if their acceleration over two timespans $t$ and $t+1$ is greater than a defined threshold $\theta$. We set $\theta$ to the acceleration average over $T, \theta=\frac{1}{T} \sum_{t=1}^{T} \frac{1}{\left|V^{\prime \prime}\right|} \sum_{i, j} \operatorname{acceleration}\left(w_{i}, w_{j}\right), w_{i}$ and $w_{j}$ are belonging $V^{\prime \prime}$.

\section{EXPERIMENTS}

To analyse the computational history in the domain of Machine Learning, we evaluate our proposed approach on a time-stamped text corpora extracted from NIPS publications. NIPS has been chosen as one of the top Machine Learning conferences in the world that covers topics from deep learning and computer vision to cognitive science and reinforcement learning. We demonstrate that our approach finds acceleration between trending keywords over time. This allows us to track the evolving scientific discovery in the field by following dynamic embeddings. The dynamic embedding is used to define the acceleration of various keywords over subsequent timespans in order to detect fast emerging keywords and subsequently emerging trends.

\section{A. NIPS Dataset and Preprocessing}

The data used for this analysis is a set of 5991 papers published between 1987 and 2015. The dataset is publicly available on Kaggle ${ }^{1}$ and contains information about papers, authors and the relation between papers and authors.

The data set was first preprocessed. Data preprocessing consists of two steps: (i) the removal of stop words from the text using Stanford NLP stop word lis ${ }^{2}$ and a list of 170 academic stop words that we defined from common

${ }^{1}$ https://www.kaggle.com/benhamner/nips-2015-papers/data

2 https://github.com/stanfordnlp/CoreNLP/blob/master/data/edu/stanford/ nlp/patterns/surface/stopwords.txt 
academic vocabulary like "introduction, abstract, table, figure, etc."; and (ii) the construction of bag of words where words are either unigrams used for standard word2vec training or bigrams extracted with word2phrase. Word2phrase attempts to learn phrases by progressively joining adjacent pairs of words with a ', character [8]. It is used as a method for corpus augmentation.

To study the temporal evolution of the trends in Machine Learning by tracking emerging scientific keywords, we divided the NIPS publications between 1987 and 2015 into six 5years timespan; however, the last timespan is 4 -years long. Therefore, the skip-gram embeddings of the year $t^{\prime}$ will contain a snapshot of the interactions between keywords in the timespan $\left(t^{\prime}-4, t^{\prime}\right)$. For instance, an embedding of the year 2005 will describe how the word embeddings of keywords developed in the years 2001 to 2005. The length of the timespan is based on the study performed by Hoonlor et al. [1] on evolving Computer Science research. That investigation showed that the average length of the evolutionary chain is 4.5 years. This choice was also tested successfully by Salatino $e t$ al. [5]. The statistics of the dataset are given in Table I]

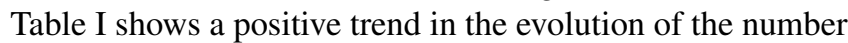
of papers per 5-years over the 1987-2015 study period. The average 5 -annual growth rate is $22 \%$, rising to $29.71 \%$ in the timespan 2007-2011.

TABLE I: Statistics of NIPS dataset

\begin{tabular}{llll}
\hline Timespan & $\sharp$ Papers & $\sharp$ Words & $\sharp$ Vocabulary \\
\hline From 1987 to 1991 & 571 & 859293 & 10823 \\
From 1992 to 1996 & 729 & 1096455 & 12651 \\
From 1997 to 2001 & 800 & 1301492 & 13471 \\
From 2002 to 2006 & 1023 & 2020697 & 16493 \\
From 2007 to 2011 & 1327 & 3243526 & 21074 \\
From 2012 to 2015 & 1541 & 4002513 & 24299 \\
\hline
\end{tabular}

\section{B. Results and Discussion}

We evaluate the use of dynamic word embeddings on the content analysis of Machine Learning scientific publications and their impact on the evolution of the main streams of Machine Learning keywords. To do so, NIPS publications published between 1987 and 2015 and divided into six 5-years timespans, have been used.

Our goal of these experiments is two-fold.

1) We aim to evaluate whether our training data with dynamic word embedding representations derived from word2vec skip-gram model (where word is not only a unigram, but can also be a bigram) is useful for tracking trends and emerging keywords for the Machine Learning domain.

2) We want to study the concordance between our deep learning trend analysis method, and the citation-based analysis method, commonly used in the literature.

To generate embeddings, we started with a text analysis step. For each timespan $t$, we created a corpus $P_{t}$ of all publications published during this time period. Then, after removing stop words, we performed term frequency statistics

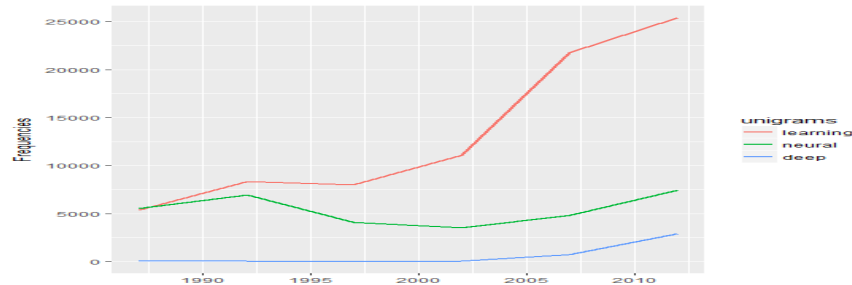

Fig. 2: Frequencies of "deep", "neural" and "learning" over time

on unique words of the vocabulary based on two types of bag-of-words: unigrams and bigrams, in order to study the evolving keywords over time based on their frequencies. Early findings [12] illustrated that word frequency itself is correlated with the success of the keywords historically. We explored the relation between dynamism and frequency change in order to gain insights into emerging keywords in the area of Machine Learning.

By examining the 20 most frequent unigrams and bigrams from the NIPS publications over the six timespans covering a total of 28 years, it was clear that the frequencies of $n$ grams evolve considerably over time. Interestingly, we found that the frequencies of some words (unigrams and bigrams) increase by approximately the same rate in specific timespans. For example, we found that the frequencies of "neural" and "learning" rose simultaneously between the timespan (1987-1991) and the timespan (1992-1996); this indicates that learning in this time period primarily relied on neural computation. Interestingly, this observation is justified by bigrams. For instance, we noticed increasing frequencies of "neuralnetworks", "reinforcement-learning" and "machine-learning" in the next timespans (1997-2001) and (2007-2011).

It seems that as the word usage increases together, these words merge and become emerging keywords. To test the effectiveness of this observation, and assuming that "deeplearning" is the emerging trend or keyword in the area of Machine Learning in the last few years while our dataset is limited to only 2015 publications, we tried to investigate an intriguing prediction based on the frequencies that we have. Considering that "deep learning" is learning based on neural computation, we tracked the frequencies of "learning", "neural" and "deep" over time. Recall that "deep" and "deep learning" do not appear on top-100 words in all timespans. We notice that suddenly the frequency of "deep" had a jump in the timespan (2012-2015) that presents the period of emergence of deep learning. Fig. 2 shows how "deep learning” as neural learning appears over this 28-year period. The frequency of "deep" remained steady, basically null (equal to 19 on the timespan 1997-2001) until 2005 where it started to rise slightly. Then, it rose dramatically to 2913 in the timespan 2012-2015. In this last time period, the frequencies of the three unigrams rose in a parallel way which justifies their concordance.

1) Qualitative results: We show that our approach results in understandable word embedding trajectories on NIPS cor- 
pora. We can automatically detect keywords that accelerate significantly to get close over time.

Fig. 3 shows t-SNE representations of the six timespans considering bag-of-unigrams, while Fig. 4 shows t-SNE representations of the last four timespans considering bag-of-bigrams. t-SNE takes the 200 dimensions via word2 vec vectors, then reduces them down to 2-dimensional $(x, y)$ coordinate pairs. The idea is to keep similar words close together on the plane, while maximising the distance between dissimilar words (words are unigrams or bigrams). We plotted the 2-D t-SNE projection of each unigram's and bigram's temporal embedding across time. For visualisation purposes, we plotted t-SNE representations of only top-100 and top-20 most frequent unigrams and bigrams respectively.

We pick 2 unigrams of interest in the t-SNE representations related to unigrams: "neural" and "learning". In all visualisations, the embeddings illustrate significant acceleration between the two unigrams. As a matter of fact, their similarity (cosine similarity) is increasing over time. For instance, it increased from 0.0657 in the second timespan (1992-1992) to 0.2235 in the fifth timespan (2007-2011). Table [II shows an increase of $70 \%$ in similarity, which suggests that learning was increasingly based on neural computation/networks and consequently the combination of these unigrams could lead to emerging keywords.

TABLE II: Temporal similarity between "neural" and "learning"

\begin{tabular}{llllll}
\hline $87-91$ & $92-96$ & $97-01$ & $02-06$ & $07-11$ & $12-15$ \\
\hline 0.1657 & 0.0657 & 0.09650 & 0.1806 & $\mathbf{0 . 2 2 3 5}$ & 0.1994 \\
\hline
\end{tabular}

Knowing that the unigram "deep" is used together with the semantics of neural computation/networks and considering that "deep" is not represented in top-100 frequent unigrams, we computed the similarity between "deep" and "learning" to verify if "deep" and "neural" get similarly close to "learning" over time. Table III shows that like "neural", "deep" gets close to "learning" chronologically; in fact, it gets even closer to "learning" with a similarity consistently higher than that of "neural" over all the timespans. These findings from the embeddings agree with the statistics previously calculated on term frequencies and support the effectiveness of our approach.

TABLE III: Temporal similarity between "deep" and "learning"

\begin{tabular}{llllll}
\hline $87-91$ & $92-96$ & $97-01$ & $02-06$ & $07-11$ & $12-15$ \\
\hline 0.2285 & 0.1914 & 0.1286 & 0.1885 & $\mathbf{0 . 2 5 6 9}$ & 0.2458 \\
\hline
\end{tabular}

Fig. 4 shows t-SNE representations of the last four timespans considering bag-of-bigrams. We plotted the 2-D t-SNE projection of each bigram's temporal embedding across time. For visualisation purposes, we plotted t-SNE representations of only top-20 most frequent bigrams.

To be consistent, we analyse t-SNE visualisations for bigrams by choosing bigrams similar to the unigrams of interest. The bigrams of interest are: "neural- networks", "neural-computation", "reinforcement-learning" and "machine-learning". As we plotted only the top-20 bigrams, not all the selected bigrams appear in all visualisations. Hence, we focus only on visualisations of the last four timespans as they mostly contain the bigrams of interest. In Fig. 4(a) (third timespan: 1997-2001), we see the bigram "reinforcement-learning" and its neighborhood derived from "neural". i.e. "neural-network", "neuralnetworks" and "neural-computation". This is: (i) semantically significant as "reinforcement-learning" by definition is called neuro-dynamic programming and needs incremental "neural networks"; and (ii) proved by similarity as the latter reaches its peak by 0.9998 during this time period. Likewise, the similarity between "machine-learning" and "neuralnetworks" peaks at almost the same value of 0.9997 while "machine-learning" is not represented in the figure that shows only the 20 most frequent bigrams. This also indicates that "reinforcement-learning" was used as "machinelearning" during this time period; in fact, the similarity between "reinforcement-learning" and "machine-learning" is equal to 0.9994 .

One timespan later (2002-2006) (Fig. 4(b)), "machinelearning" appears and its similarity to "neural-networks" drops significantly to 0.8686 . This shows that "machine learning" started to flourish towards the end of 1990s as an independent topic while "reinforcement learning" remained linked to "neural computation/networks".

In the fifth timespan (2007-2011) (Fig. 4(c) "neuralnetworks/computation" does not appear in the top-20 frequent bigrams. However, this timespan highlights the reapproximation between "machine learning" and "reinforcement learning" that incorporates "neural networks". This is insightful as it shows how "machine learning" is increasingly related to "neural networks/computation".

The last timespan (2012-2015) (Fig. 4(d) also shows that "machine learning" is geometrically very close to "neural networks" that re-appeared, while "reinforcement learning" disappeared from the top-20 bigrams. This shows that possibly "machine learning" increasingly implies "neural networks" just as "reinforcement learning” did earlier.

Based on the findings of bigrams embedding and knowing that "deep-learning" was the emerging keyword in the last few years, we computed the similarity between "machinelearning" and "deep-learning" and we found that it is equal to 0.8716 while "deep-learning" does not exist in previous timespan-vocabularies. Consequently, we can assume that "deep-learning" is the keyword that emerged from the convergence between "machine-learning" and "neural-networks".

Our qualitative and quantitative analyses on both unigrams and bigrams show that the embeddings we learned reveal interesting patterns in the similarity between potentially emerging keywords over time. To prove that, we created similarity matrices as described in section II-C for the top-20 frequent and overlapped bigrams and only a couple of unigrams \{ "neural", "learning" $\}$ in order to be consistent to the unigrams and bigrams of interest previously picked. The similarity matrices 


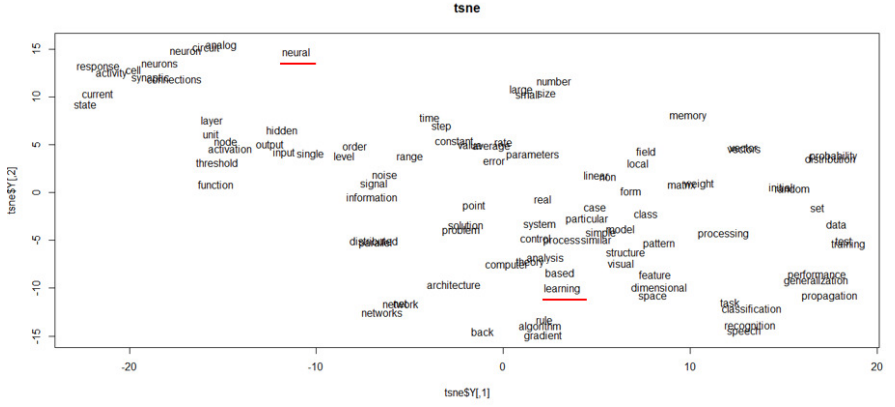

(a) 1987-1991

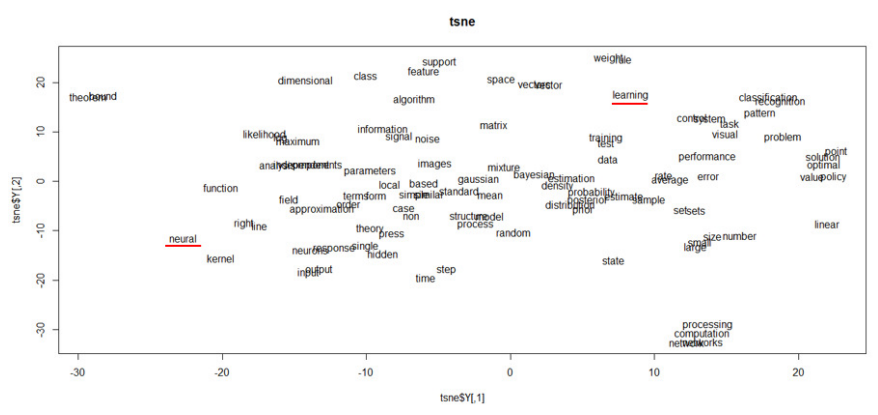

(c) $1997-2001$

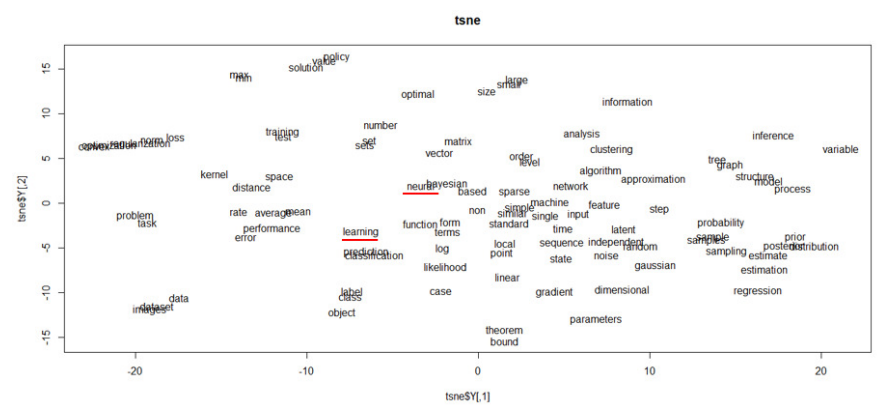

(e) $2007-2011$

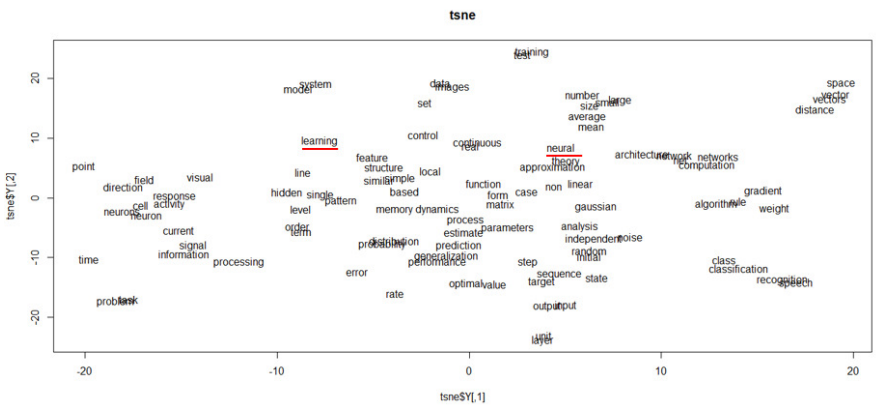

(b) $1992-1996$

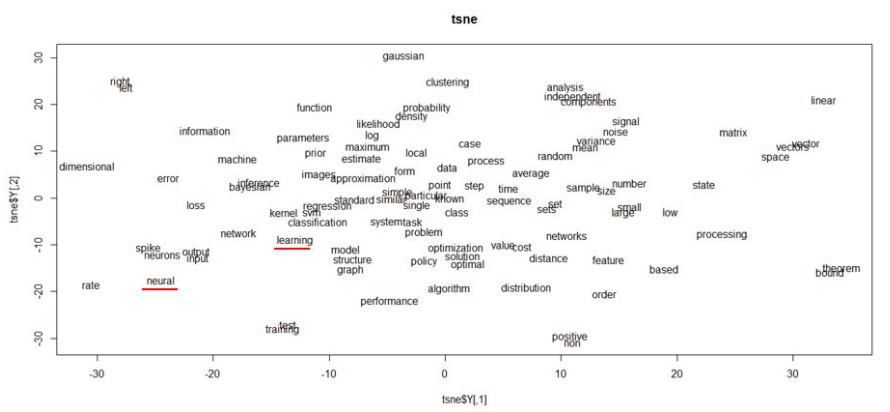

(d) $2002-2006$

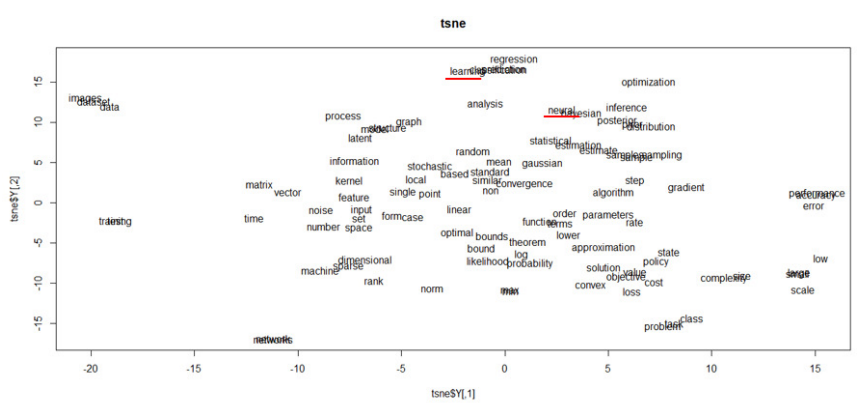

(f) $2012-2015$

Fig. 3: t-SNE of top 100 unigrams of all timespans; the overlapping keywords - horizontally from left to right, and then vertically from top to bottom - are as follows: in 3(a) \{ "distributed, parallel", "probability, distribution", "initial, random"\}, in 3(b) \{ "problem, task", "distribution, probability", "unit, layer"\}, in 3(c) \{ "theorem, bound", "analysis, independant", "simple, similar"\}, in 3(e) \{ "optimization, normalization", "dataset, images", "prediction, classification", "posterior, distribution"\}, and in 3(f) [ "training, testing", "model, architecture", "prediction, classification", "min, max", "problem, task", "performance, accuracy", "large, small"\}

contain the cosine similarity between the embedding vectors of every two keywords.

After creating the similarity matrices, we generated the acceleration matrix as described in II-C. We computed the average acceleration $\theta$ that corresponds to the average over all the selected keywords. $\theta$ was negative and equal to -0.0656 . Overall, almost all accelerations are negative but some of them were speeding up. For instance, the couple of bigrams \{ "neural-networks", "reinforcement-learning" $\}$ have an acceleration of -0.011 , which is much greater than the average $\theta$. Interestingly, we found that the acceleration of the couple of bigrams \{ "neural-networks", "machine-learning"\} is positive and equal to 0.0094 . Respectively, the acceleration of the couple of unigrams $\{$ "neural", "learning" is positive and has a value of 0.02 . Both of them have an acceleration much greater than the average $(\theta)$. These findings support the previous ones and show that neural-based learning was speeding up over time. Similar to previous investigations about the emergence of "deep learning", we computed the acceleration of two unigrams \{ "deep", "learning" $\}$ and two bigrams \{ "neural-network", "deep-learning"\}. Their values are respectively 0.0034 and 0.1649 , showing a substantial speed up over the average $\theta$.

2) Quantitative results: In order to test effectiveness of the proposed DeepHist in detecting emerging keywords in the area of Machine Learning, we validate it with the citation counting approach which is widely used in the literature and provides a snapshot of a fast-growing field. The objective is to check 


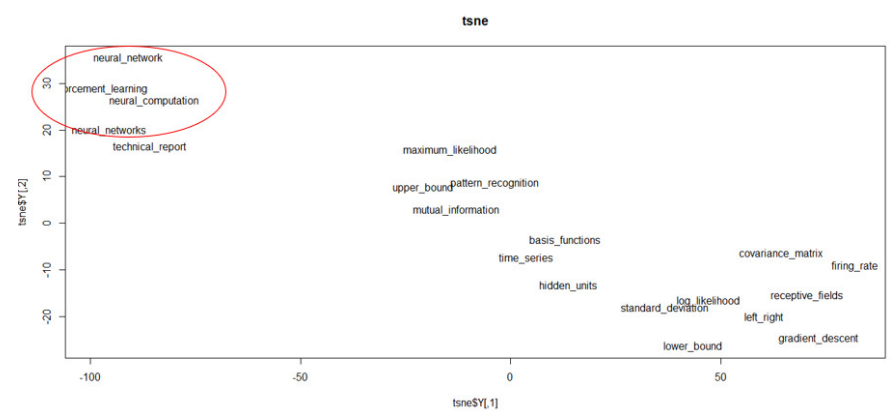

(a) $1997-2001$

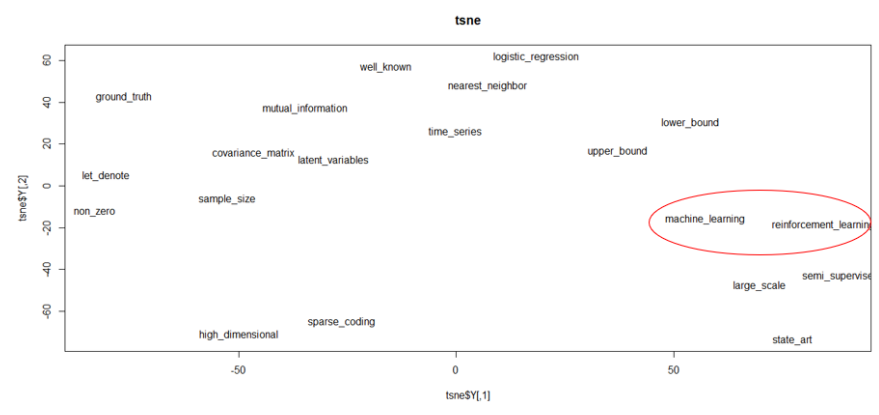

(c) $2007-2011$

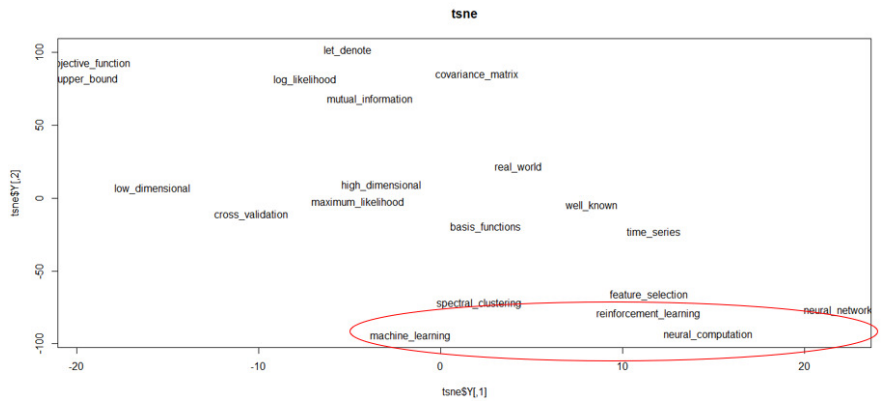

(b) $2002-2006$

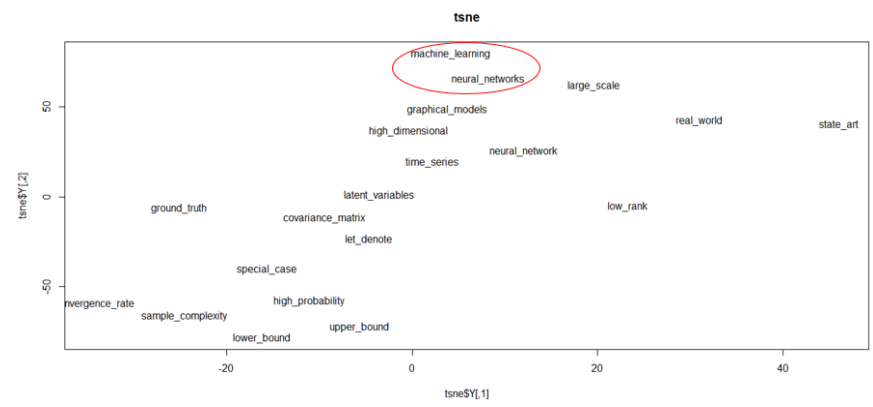

(d) $2012-2015$

Fig. 4: t-SNE of top 20 bigrams of the four timespans between 1997 and 2015

the extent to which citation analysis supports the findings of DeepHist. To do so, we retrieved academic citations of all the NIPS publications in our dataset (1987 to 2015) using the Public or Perish software ${ }^{3}$ that uses Google Scholar ${ }^{4}$ to obtain the raw citations.

For consistency, we tracked the citation counts of publications with previously selected frequent keywords (the keywords of interest we picked in the qualitative analysis) over time such that in each timespan we noted the citation counts of the publications that used the picked keywords in their titles; we assume that the title plays a pivotal role in communicating research.

We compare the acceleration of citation counts of publications mentioning the keywords of interest in their titles with the acceleration of similarities of these keywords that our embeddings return over all timespans. Spearman's correlation coefficient $\rho$ has been used to measure the strength and direction of association between these two variables. $\rho$ is defined as following:

$$
\rho=\frac{\sum_{s}\left(x_{s}-\bar{x}\right)\left(y_{s}-\bar{y}\right)}{\sqrt{\sum_{s}\left(x_{s}-\bar{x}\right)^{2} \sum_{s}\left(y_{s}-\bar{y}\right)^{2}}}
$$

where $s$ is the paired score (citation_count, similarity), $x$ and $y$ correspond to the citations counts and similarity values, $\bar{x}$ and $\bar{y}$ correspond respectively to the mean of citations counts and the mean of similarity values.

\footnotetext{
3 https://harzing.com/resources/publish-or-perish ${ }^{4}$ https://scholar.google.com/
}

Spearman's correlation coefficient has been computed for all the pairs of picked keywords. Interestingly, we found that $100 \%$ of cases have a positive correlation with an average of 0.422 . $67 \%$ of these correlations are strong with $\rho$ coefficient greater than 0.6 .

Fig. 5(a) and Fig. 5(b) show the relationships between the citation counts and the similarities of the keywords of interest "machine-learning - neural-networks $(M L-N N)$ " and "neural-networks - deep-learning $(N N-D L)$ " respectively. Fig. 5(a) has Spearman's correlation coefficient $\rho$ equal to 0.2 . If we do not consider the last point where the similarity between "machine-learning" and "neural-networks" dropped in the timespan (2002-2006), $\rho$ coefficient is much higher and equal to 0.9 . This observation could be justified by the fact that "machine learning" started to flourish towards the end of 1990s as an independent topic which justifies the decrease in similarity with "neural-networks". Overall, this new finding confirms our previous findings stating that learning was correlated to neural networks over time. Fig. 5(b) has $\rho$ coefficient equal to 0.654 . This result perfectly matches with our previous findings where the citation count was slightly small in the first four timespans. Then, suddenly it rose dramatically to reach 3223 in the last timespan (20122015). A significant rise of these citation counts is clearly seen which goes with the increase in the similarity and the acceleration previously detailed, and shows that "learning" was increasingly relying on "neural networks". The emerging keyword "deep-learning" goes in parallel with the keywords of interest "neural-networks" and "machine-learning" and dramatically increased in the last timespan, which supports 
the assumption that "deep-learning" is now the trend.

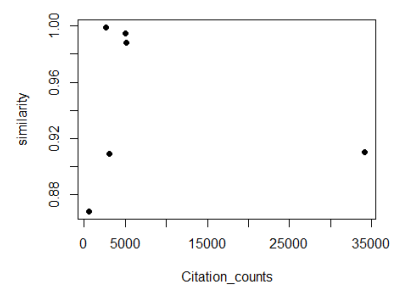

(a) $\mathrm{ML}-\mathrm{NN}$

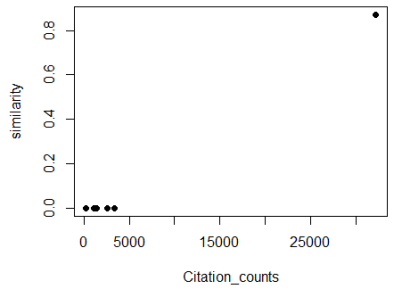

(b) $\mathrm{NN}-\mathrm{DL}$
Fig. 5: Spearman's coefficient plot of $(\mathrm{ML}-\mathrm{NN})$ and $(\mathrm{NN}-$ DL)

These findings resulting from citation counts support the effectiveness of our approach based on dynamic word embeddings in discovering rising keywords in the domain of Machine Learning.

\section{RELATED WORK}

The emerging research addressing trend analysis can be categorized into three categories: bibliometrics-based approaches [13] that are based on social network analysis and citation analysis; content-based approaches [14] that treat entities - essentially keywords - reflecting the paper content [14]; and hybrid approaches [15] that combine both citation and content. These approaches dealing with trend analysis have been applied to a wide range of disciplines such as business [16], computer science [1], [15], etc. In this paper, we concentrate on an area within Computer Science (CS).

While we are not aware of previous works on predicting research trends in CS by drilling into paper content and following a fine-grained content analysis, there are few works addressing related research problems in investigating general publication trends, citation trends and evolution of research areas following a coarse-grained analysis. For instance, Hoonlor et al. [1] analysed data on proposals for grants supported by the U.S National Foundation and on CS publications in the ACM Digital Library and IEEE Xplore Digital Library using sequence mining and bursty word detection. Similarly, Hou et $a l$. revealed the evolution of research topics between 2009 and 2016 using the timeline knowledge map through Document-Citation Analysis (DCA). In the same context, Effendy and Yap [15] performed trend analysis using the Microsoft Academic Graph (MAG) dataset. Both the above approaches to trend analysis in CS focus on citation analysis which fails to dig into the paper content and takes time to reveal trends.

\section{CONCLUSIONS}

We offer DeepHist, a deep learning-based approach for computational history and apply it to the NIPS publications to produce insights about the field of Machine Learning and track the evolution of new trends.

This work addressed this challenge in an innovative way by bringing together qualitative and quantitative analysis of NIPS publications during the time period 1987-2015. Both analyses drilled into the paper content by computing and visualising temporal keyword embeddings over six 5-years timespans. We explored the similarity between keywords by embedding vectors to create a similarity matrix of frequent keywords. Then, based on this matrix we created an acceleration matrix that reports the acceleration between keywords over time in order to capture the speeding up keywords that may result in a trending keyword. Our results were able to detect that "deeplearning" was the convergence between "machine-learning" and "neural-networks". Our approach has been validated against citation count analysis, and its effectiveness has been demonstrated.

As future work, we plan to generalise our approach on different research areas such as physics, biology or medicine where it would be interesting to see whether a novel treatment or a certain combination of drugs for cancer is beginning to rise. Furthermore, we plan to expand the embedding technique with more text analysis techniques that explore the semantics of paper content and help to detect emerging topics such as topic modeling.

\section{REFERENCES}

[1] A. Hoonlor, B. K. Szymanski, and M. J. Zaki, "Trends in computer science research," Commun. ACM, vol. 56, no. 10, pp. 74-83, 2013.

[2] L. Bornmann and H. Daniel, "What do citation counts measure? a review of studies on citing behavior," Documentation, vol. 64, no. 1, pp. 45-80, 2008.

[3] R. Dey, A. Roy, T. Chakraborty, and S. Ghosh, "Sleeping beauties in computer science: characterization and early identification," Scientometrics, vol. 113, no. 3, pp. 1645-1663, 2017.

[4] A. Anderson, D. McFarland, and D. Jurafsky, "Towards a computational history of the acl: 1980-2008," in ACL-2012 Special Workshop on Rediscovering 50 Years of Discoveries, 2012, pp. 13-21.

[5] A. A. Salatino, F. Osborne, and E. Motta, "How are topics born? understanding the research dynamics preceding the emergence of new areas," PeerJ Computer Science, vol. 3, p. e119, 2017.

[6] T. Mikolov, W.-t. Yih, and G. Zweig, "Linguistic regularities in continuous space word representations." in NAACL-HLT, 2013, pp. 746-751.

[7] L. van der Maaten and G. Hinton, "Visualizing data using t-SNE," Journal of Machine Learning Research, vol. 9, pp. 2579-2605, 2008.

[8] T. Mikolov, I. Sutskever, K. Chen, G. S. Corrado, and J. Dean, "Distributed representations of words and phrases and their compositionality," in NIPS, 2013, pp. 3111-3119.

[9] A. Dridi, M. M. Gaber, R. M. A. Azad, and J. Bhogal, "k-nn embedding stability for word2vec hyper-parametrisation in scientific text," in Discovery Science, 2018, pp. 328-343.

[10] A. Rinaldo, A. Singh, R. Nugent, and L. Wasserman, "Stability of density-based clustering," Journal of Machine Learning Research, vol. 13, no. 1, pp. 905-948, 2012.

[11] Nicolai Meinshausen and Peter Bhlmann, "Stability selection," Royal Statistical Society, vol. 72, no. 4, pp. 417-473, 2010.

[12] E. Lieberman, J.-B. Michel, J. Jackson, T. Tang, and M. A. Nowak, "Quantifying the evolutionary dynamics of language," Nature, vol. 449, no. 7163, pp. 713-716, 2007.

[13] J. Hou, X. Yang, and C. Chen, "Emerging trends and new developments in information science: a document co-citation analysis (2009-2016)," Scientometrics, vol. 115, no. 2, pp. 869-892, 2018.

[14] C. Weismayer and I. Pezenka, "Identifying emerging research fields: a longitudinal latent semantic keyword analysis," Scientometrics, vol. 113, no. 3, pp. 1757-1785, 2017.

[15] S. Effendy and R. H. Yap, "Analysing trends in computer science research: A preliminary study using the microsoft academic graph," in $W W W, 2017$, pp. 1245-1250.

[16] D. E. Rossetto, R. C. Bernardes, F. M. Borini, and C. C. Gattaz, "Structure and evolution of innovation research in the last 60 years: review and future trends in the field of business through the citations and co-citations analysis," Scientometrics, 2018. 